\title{
Ultra High Energetic Cosmic Ray and Neutrino Observations with LOFAR and SKA
}

\author{
Maaijke Mevius $^{* a}$, Olaf Scholten ${ }^{a}$ and Clancy W. James ${ }^{b}$ \\ for the LOFAR CR and NuMoon Collaboration \\ ${ }^{a}$ Kernfysisch Versneller Instituut, University of Groningen,9747 AA, Groningen \\ ${ }^{b}$ Department of Astrophysics, IMAPP, Radboud University, 6500 GL, Nijmegen \\ E-mail: meviusakvi.nl
}

\begin{abstract}
The lunar Cherenkov technique is a method to detect ultra-high-energy (UHE) cosmic-rays and neutrinos with Earth-based radio-telescopes. When an UHE particle hits the Moon, it initiates a hadronic cascade below the surface. The cascade has a negative charge excess that propagates faster than the local speed of light in the medium, producing a short pulse of radio Cherenkov emission. The NuMoon project aims to detect these lunar radio pulses on Earth with lowfrequency radio telescopes. In the first phase a measurement was performed with the Westerbork Synthesis Radio Telescope array, resulting in an upper limit on the neutrino flux an order of magnitude better than previous existing limits. We are currently preparing to perform this measurement with LOFAR. The expected sensitivity of LOFAR reaches flux limits within the range of some theoretical production models. With SKA it should be possible to detect neutrinos that are the decay products of pions produced in the interaction of UHE cosmic particles with the cosmic microwave background (GZK neutrinos).
\end{abstract}

ISKAF2010 Science Meeting

June $10-142010$

Assen, the Netherlands

\footnotetext{
* Speaker.
} 


\section{Ultra-high-energy cosmic rays to radio telescopes}

The origin of the highest-energy particles in nature, the ultra-high-energy cosmic rays, remains unknown. The production mechanism of these energetic nuclei is unidentified primarily because, being charged, their trajectories are bent in cosmic magnetic fields. At extremely high energies, the bending is small enough that a correlation of arrival directions with extragalactic objects has been detected [1]. However, here the flux is of order one per $\mathrm{km}^{2}$ per century, so that even the largest detectors (of $3000 \mathrm{~km}^{2}$ effective area) are too small. Thus even larger detectors such as Auger North [2] and JEM-EUSO [3] of up to $100,000 \mathrm{~km}^{2}$ effective area have been proposed to increase our knowledge of the source of these particles.

An alternative approach is to observe ultra-high energy neutrinos. 'Cosmogenic' neutrinos are expected from interactions of the highest-energy cosmic rays with background photon fields, in particular the $\mathrm{CMB}$, since protons above $\sim 5 \times 10^{19} \mathrm{eV}$ see these background photons as gammarays sufficient for pion photo-production. The resulting energy loss of the cosmic rays is known as

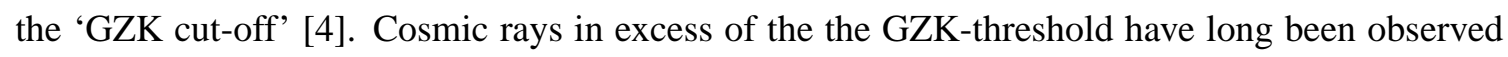
[5], 6], and the flux falls sharply near the GZK cut-off in a manner consistent with such a cut-off [7], so that a flux of UHE neutrinos is almost guaranteed. Since neutrinos are uncharged and rarely interact, they will travel directly from their source to an observer unimpeded and undeflected. Major experiments searching for such neutrinos include IceCube, RICE, ANITA, and the Pierre Auger experiment. However, while the UHE neutrino flux is predicted to be larger than that for UHE cosmic rays, they are nonetheless rare, and since they interact rarely (penetrating of order $100 \mathrm{~km}$ of water), a large volume detector is also required.

Directly implementing large areas/volumes for UHE particle detection is prohibitively expensive, and thus a remote-detection method must be devised. In this contribution, we discuss the lunar Cherenkov technique, by which ground-based radio-telescopes observe the Moon to search for UHE particle interactions in its outer layers [8].

\section{Principle of detection using the Moon}

In the interaction of an UHE neutrino with the material in the lunar regolith (the upper layer of dusty Lunar 'soil'), about $20 \%$ of its energy is converted into a hadronic shower consisting of large numbers of energetic hadrons, leptons and photons all moving through the regolith with the light velocity. For a UHE cosmic ray, $100 \%$ of its energy is converted into a hadronic shower. Due to scattering processes and positron annihilation with regolith electrons, a net excess of electrons is formed in the shower, resulting in a charge excess that moves with a velocity larger than that of light in the medium, and, therefore, in the emission of Cherenkov radiation. The typical width of the charge cloud is $\sim 10 \mathrm{~cm}$. At wavelengths larger than this typical size (i.e. frequencies of about $3 \mathrm{GHz}$ and below), the emitted Cherenkov radiation is coherent, a process known as the Askaryan effect [9]. The emitted Cherenkov radiation can be observed with sensitive radio telescopes on Earth.

The maximum intensity of the coherent Cherenkov emission is reached at the a frequency of about $3 \mathrm{GHz}$, where the emitted radiation is concentrated in a narrow cone around the Cherenkov angle. The intensity drops at lower frequencies, but the angular spread of the emission increases. 
For a shower parallel to the surface of the Moon, the Cherenkov angle corresponds to that of total internal reflection. While UHE neutrinos may penetrate the Moon, interact as 'up-coming' events, and allow high-frequency radiation from the Cherenkov angle to escape, cosmic rays will always interact immediately. Therefore, for high frequencies, only the few cosmic rays that skim the rim of the Moon, directed towards Earth, can be detected. For lower frequencies (between 100 and 200 $\mathrm{MHz}$ ), even at large incoming cosmic-ray angles the radiation can escape the surface of the Moon, and as a result, the total surface of the Moon may emit detectable signals [10]. A major advantage of using the Moon for the detection of UHE neutrinos, is the rather long attenuation length of $\lambda_{r}=9 \mathrm{~m} / v[G H z]$ for radio waves, which makes a very large detection volume. As a result the detection efficiency increases with about the third power of the wavelength. A trade-off for the larger detection probability at low frequencies is the lower intensity of the signal and therefore the loss of sensitivity at lower energies.

The idea of using the Moon as a detecting volume to measure cosmic rays was first proposed by Dagkesamamnskii and Zheleznykh[8], while the first experiments were carried out with the Parkes telescope [11] and subsequently at Goldstone (GLUE) [12], Kalyazin [13], ATCA [14] and Westerbork [15]. Of these, the first are all performed at relatively high frequencies $(2 \mathrm{GHz})$. We will discuss the Westerbork measurement in more detail in the next section. Although none of these experiments have recorded any signal, simulations indicate that next-generation telescopes, such as SKA, could probe the flux of UHE neutrinos and will likely be sensitive to the currently observed flux of UHE cosmic rays. Currently, an initiative to perform the UHE neutrino measurement using the lunar Cherenkov technique at low frequencies with the LOFAR radio telescope is under development. This will be discussed in section 4 .

\section{WSRT Observations}

The emission of $3 \mathrm{~m}$ radio waves from impacts of high energy neutrinos on the Moon is exploited in our observations with the Westerbork Synthesis Radio Telescope (WSRT) and has resulted in the most stringent flux limit at the highest energies [15]. The WSRT consists of an array of 14 parabolic antennas of $25 \mathrm{~m}$ diameter. Only 11 of the 12 equally spaced WSRT dishes were used for this experiment. In the observations we employed the Low Frequency Front Ends (LFFEs) which cover the frequency range $115-180 \mathrm{MHz}$ with full polarisation sensitivity, sampled as 8 sub-bands of $20 \mathrm{MHz}$ each by the Pulsar Machine II (PuMa II) backend [16]. We used 4 frequency bands centered at frequencies of $123,137,151$ and $165 \mathrm{MHz}$ for each of two different beams aimed at different sides of the Moon. This created the possibility of an anti-coincidence trigger since a lunar Cherenkov pulse should only be visible in only one of the two beams. The total bandwidth per beam was $65 \mathrm{MHz}$. The time series data was recorded for each sub-band with a sampling frequency of $40 \mathrm{MHz}$. The data was processed in blocks of $0.1 \mathrm{~s}$, where each block was divided in 200 traces of 20,000 time samples.

In an effective observation time of $40 \mathrm{~h}$ no pulses were observed, resulting in the $90 \%$ confidence upper limit on the neutrino flux shown in figure 11. The limit is almost an order of magnitude better than previous limits in the UHE region set by ANITA [17] and FORTE [18]. 


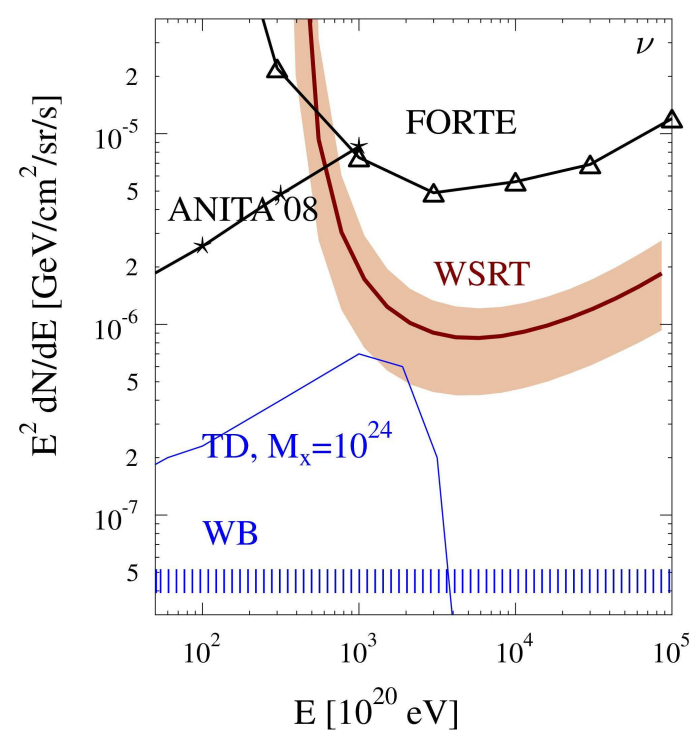

Figure 1: The limit [15] on the flux of UHE neutrinos obtained from observations with the WSRT is compared with those of other experiments and some model calculations, see text.

\section{LOFAR}

We are currently setting up an experiment with the high-band antennas (100-200 MHz) of the LOFAR radio-telescope. LOFAR provides many advantages over WSRT. Not only can a higher sensitivity be reached due to the larger collecting area $(\sim 3$ times that of WSRT for the core of LOFAR), but also the pointing accuracy will allow us to determine the location of an event position on the Moon with much better precision, important for RFI discrimination. The multi-beam possibility allows for the formation of sufficiently many coherent beams to cover the complete lunar surface. A third advantage are the Transient Buffer Boards. These will store the raw antenna data for a short time, which allows the implementation of an advanced self trigger algorithm. Furthermore, once a trigger is issued, the full resolution, full-bandwidth time-series data can be stored for off-line analysis.

We are currently developing the trigger algorithm, in order to considerably reduce the raw data rate of $\sim 1 \mathrm{~Tb} / \mathrm{s}$. For the trigger itself, only data from the core stations will be considered. About half the bandwidth of the station data will be send to the Blue-Gene/P supercomputer in Groningen, where the data will be coherently added to form $\sim 50$ beams to cover the full surface of the Moon. A short time (few ns) peak will be searched for in the time-series data, with the requirement that a peak will only be visible in one or few of the beams. Once a trigger is issued, $\sim 1 \mathrm{~ms}$ of raw data from all stations will be written to disk for off-line analysis.

An important issue, especially at low frequencies, is the frequency-dependent dispersive effect of the ionosphere. At LOFAR-frequencies a typical ionospheric electron content of $10 \mathrm{TECU}$ causes a dispersion which spreads the original input signal of a few ns over several time-bins, as illustrated in figure 2 In order to still be able to extract the signal the data needs to be de-dispersed. To accomplish this, the absolute electron content of the ionosphere needs to be known online with an accuracy of about 1 TECU. This can be improved in the off-line analysis. Schemes to calibrate 


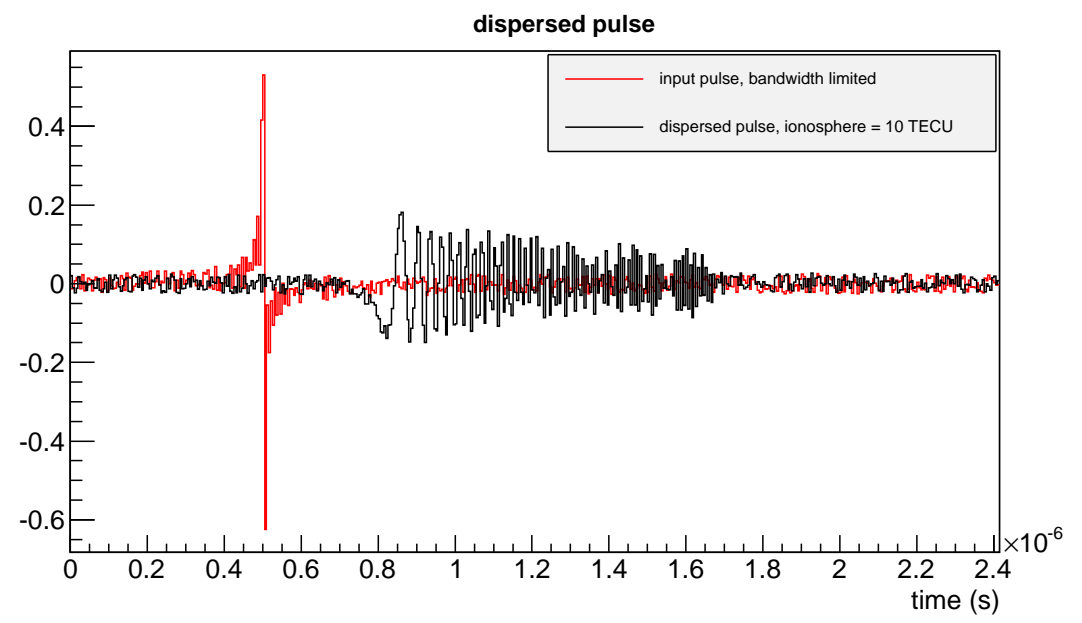

Figure 2: Time-series of a dispersed pulse, for a typical ionosphere of 10 TECunits. The original ns bandwidth limited pulse is spread over several bins due to the dispersion.

the absolute ionosphere are currently being developed.

So called transient noise, i.e. short-time noise pulses, is of major concern since it may mimic our signal. To reduce the trigger rate and therefore the dead-time of the system, it needs to be identified and ignored with good accuracy in the online trigger. In the offline analysis noise pulses need to be fully eliminated. We are investigating the properties of this noise. It is expected that the resolution of LOFAR, which will be even better when the longer baselines are included in the off-line analysis, will be sufficient to discriminate signals from the Moon from man-made noise.

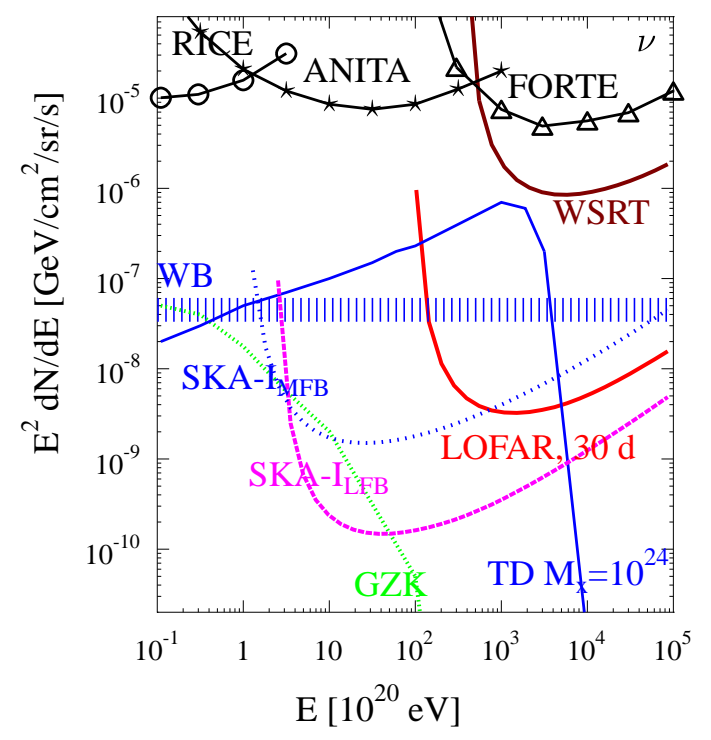

Figure 3: Expected sensitivities on the neutrino flux of LOFAR and SKA compared to earlier experimental upper limits and some models. The LOFAR sensitivity is shown for 30 days of data taking. Also shown are the expected sensitivity curves of the SKA for low $(L F B, 100-300 \mathrm{MHz})$ and intermediate frequencies (MFB, 300-500 MHz). 


\section{Outlook}

In figure 3 the neutrino flux sensitivity is indicated that can be reached with one month of observation with LOFAR. The sensitivity is such that one month of accumulated time should result in a large number of observed events for a neutrino flux at the level of the Waxman-Bahcall limit [19]. In the same figure the expected SKA sensitivities are shown, both for the low 'LFB' (100$300 \mathrm{MHz}$ band) and intermediate 'MFB' (300-500 Mhz) frequencies. Expected SKA sensitivities are such that neutrinos from decay products of GZK interactions - using the predictions by [20] can be observed.

The predicted (and promising) sensitivities of this next generation of instruments for the detection of both UHE cosmic rays and neutrinos highlight the importance of developmental work such as that we have described in section 4 with LOFAR. Harnessing distributed arrays of detectors optimised for imaging to a search for nanosecond pulses from UHE particle interactions is a great technical challenge. However, projects such as NuMoon and others have pointed the way towards accomplishing this goal, and in the near future will begin to set unprecedented limits on the flux of these elusive UHE particle - or detect a new flux and begin probing the highest-energy astrophysical phenomena.

\section{References}

[1] The Pierre Auger Collaboration (J. Abraham et al.), Science 318, 938 (2007).

[2] J.L. Harton for the Pierre Auger Collaboration, Progress with the Northern Part of the Pierre Auger Observatory, presented at the $31^{\text {st }}$ International Cosmic Ray Conference, Łòdż, Poland (2009).

[3] T. Ebisuzaki et al., Nuc. Phys. B Proc. Supp. 175, 237 (2008).

[4] K. Greisen, Phys. Rev. Lett. 16, 748 (1966); G.T. Zatsepin, V.A. Kuzmin, JETP Lett. 4, 78 (1966).

[5] D.J. Bird et al., ApJ 441, 144 (1995).

[6] M. Takeda et al., Astropart. Phys. 19, 447 (2003).

[7] The Pierre Auger Collaboration (J. Abraham et al.), Phys. Rev. Lett. 101, 061101 (2008).

[8] R. Dagesamanskii and I. Zheleznyk, Sov. Phys. J.E.T.P. 50, 233 (1989).

[9] G. Askaryan, Sov. Phys. J.E.T.P. 14, 441 (1962).

[10] O. Scholten et al., Astropart. Phys. 26, 219 (2006).

[11] T. Hankins, R. Ekers, and J. O'Sullivan, Mon. Not. Roy. Astron. Soc. 283, 1027 (1996).

[12] P. Gorham et al., Phys. Rev. Lett. 93, 41101 (2004).

[13] A. Beresnyak et al., Astron. Rep. 49, 127 (2005).

[14] C.W. James et al., Phys. Rev. D 81, 042003 (2010).

[15] O. Scholten et al., Phys. Rev. Lett. 103 (2009) 191301; S. Buitink et al., arXiv:1004.0274v1.

[16] R. Karuppusamy, B. Stappers, W. van Straten (2008), Publ. Astron. Soc. Pacific 120, (2008) 191.

[17] P. Gorham et al., Phys. Rev. Lett. 103, 051103 (2009).

[18] H. Lethinen et al., Phys. Rev. D 69, 013008 (2004).

[19] E. Waxman and J.N. Bahcall, Phys. Rev. D59, 023002 (1999).

[20] R. Engel, D. Seckel, T. Stanev, Phys. Rev. D 64, 93010 (2001). 